Procecdings of the 21st International Conferenec on Low Temperature Physics Praguc, Augusi 8-14, 1996

Part S5 - LT Properties of Solids 2: Electrons in 2D and weak localization

\title{
Even denominator filling factors in the thermoelectric power of a 2DEG
}

\author{
S.A.J. Wiegers ${ }^{a b}$, B. Tieke ${ }^{a}$, U. Zeitler ${ }^{a c}$, R. Fletcher ${ }^{d}$, A.K. Geim ${ }^{a}$, J.C. Maan ${ }^{a}$ and M. Henini ${ }^{c}$ \\ a High Field Magnet Laboratory, University of Nijmegen, 6525 ED Nijınegen, The Netherlands \\ ${ }^{b}$ Grenoble High Magnetic Field Laboratory, MPI-CNRS, BP 166, Grenoble Cedex 9, France \\ c Department of Physics, University of Nottingham, Nottingham NG7 2RD, UK \\ d Physics Department, Queen's University, Kingston, Ontario, Canada K7L 3N6
}

We have investigated the interaction of phonons with a 2DEG in the FQH regime with phonon drag thermoelectric power (TEP). We find that the TEP at filling factors with the same even denominator is identical and at other even denominator filling factors they differ only by a constant. Assuming these states to be Composite Fermions (CF), we can explain our observations by extending a zero magnetic field theory for phonon drag to the CF-phonon interaction. This analysis is further corroborated by the observed $\mathrm{T}^{4}$ dependence of the CF TEP.

\section{INTRODUCTION}

The fractional quantum Hall effect (FQHE) has recently been attributed to quantum oscillations of new quasi-particles, called Composite Fermions (CF) $[1,2]$. The CF consist of an electron and an even number of flux quanta. The FQHE of the electrons can be viewed as the integer quantum Hall effect of the CF's. In particular, the magnetic field for which $\nu$ equals $1 / 2$, appears as zero field for the CF's.

We have studied the interaction between phonons and CF's by thermoelectric power (TEP) measurements on the 2DEG. The measurements were carried out on a GaAs-AlGaAs heterostructure with density and mobility in the range of $1.0-1.9 \times 10^{11} \mathrm{~cm}^{-2}$ and $0.6-1.0 \times 10^{6} \mathrm{~cm}^{2} / \mathrm{Vs}$, respectively. $\mathrm{A}$ known temperature gradient was set up along the sample, which was thermally anchored to the mixing chamber of a dilution refrigerator.

\section{RESULTS}

In fig. 1 we present the relative thermopower $S_{x x} / S_{x x}(\nu=1 / 2)$ as a function of magnetic field for two temperatures. One observes that $S_{x x}(3 / 2)=S_{x x}(1 / 2)$, and that the ratio $\mathrm{S}_{x x}(3 / 4) / \mathrm{S}_{x x}(1 / 2)$ is about equal to 2.2 . This is seen for all temperatures below about $0.5 \mathrm{~K}$ where the features of the FQHE become visible. For lower electron densities, where the $\nu=1 / 4$ state becomes visible, we observe similarly that $S_{x x}(3 / 4)=S_{x x}(1 / 4)$.

In fig. 2 we display the temperature dependence of $\mathrm{S}_{x x}$ at $\nu=1 / 2$ and $3 / 2$ and at $\mathrm{B}=0$. Within experimental error, all three curves follow a $\mathrm{T}^{4}$ dependence at low temperatures. At other than even denominator filling factors the low temperature behavior of $\mathrm{S}_{x x}$ is different from a $\mathrm{T}^{4}$ law.

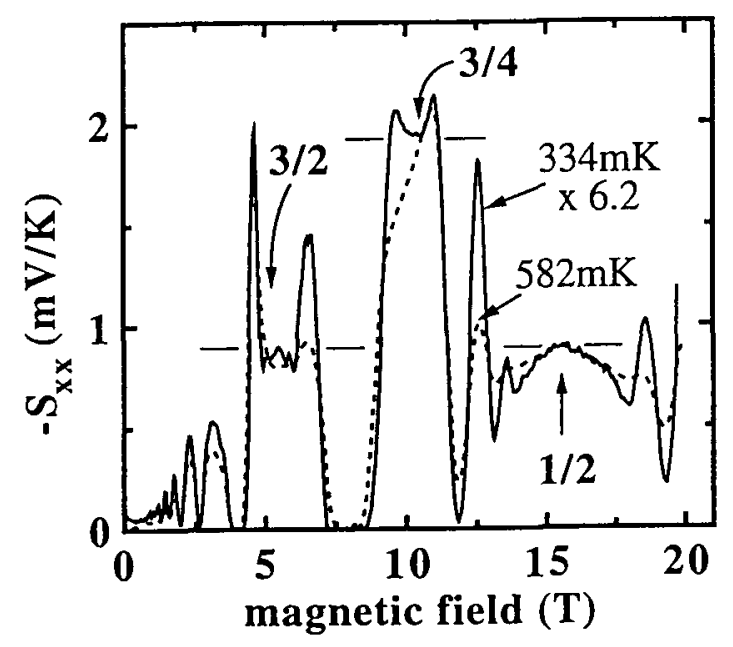

Figure 1: Examples of the magnetic field dependence of the longitudinal component $S_{x x}$ of the TEP. The horizontal lines mark the constant relative value for the even denominator filling factors $\nu=\frac{3}{2}$ and $\nu=$ $\frac{3}{4}$. 


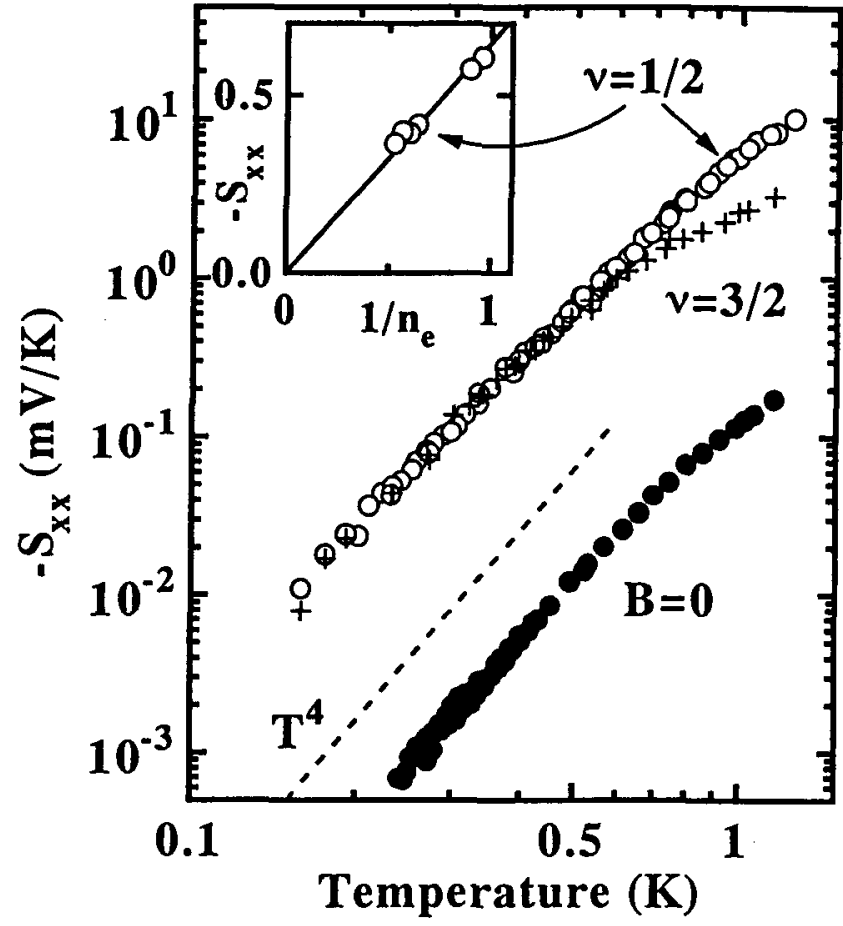

Figure 2: The temperature dependence of the absolute value of the TEP for even denominator filling factors and zero magnetic field. The dashed curve indicates a $T^{4}$ dependence. The inset shows $S_{x x}\left(\frac{1}{2}\right)$ at $T=$ $440 \mathrm{mK}$ (in $m V / K$ ) versus the inverse density $1 / n_{e}$ (in units of $10^{-15} \mathrm{~m}^{2}$ ).

\section{DISCUSSION}

We discuss first the $\mathrm{T}^{4}$ temperature dependence of $S_{x x}$ at $\nu=3 / 2$ and $1 / 2$. The similarity to the Tdependence at $\mathrm{B}=0$ shows that phonon drag is the common origin of the TEP. It suggests furthermore that for the $\nu=3 / 2$ and $1 / 2$ states we are dealing with the phonon drag TEP of a quasi-particle (CF) in a zero effective field. This fits exactly the theory of ref. [2]: at $\nu=1 / 2$ the CF's experience zero magnetic field while the $\nu=3 / 2$ state is described as a half filled Landau level to which a full Landau level is added. The experimentally observed equal values for $S_{x x}$ at $3 / 2$ and $1 / 2$ confirm that the CF-phonon interaction is unchanged when a full Landau level is added.
The expression for zero ficld phonon drag TEP derived in ref. [3] can be rewritten for the case of the CF's as [4]:

$$
S_{x x} \propto T^{4} \frac{m_{e}^{*}}{n_{e}} \iint \frac{m_{Q} v_{a}}{\hbar k_{F}^{Q}} f\left(q, q_{z}\right) d q d q_{z}
$$

where $m_{e}^{*}$ and $n_{e}$ represent the effective electron band mass and the electron density, $v_{s}$ is the velocity of sound in GaAs and $\mathrm{m}_{Q}$ and $\mathrm{k}_{F}^{Q}$ represent the $\mathrm{CF}$ effective mass and Fermi wave vector. In this equation $f\left(q, q_{z}\right)$ is a function containing the details of the scattering events, where $q$ and $q_{z}$ represent dimensionless phonon wave vectors.

Since $\mathrm{m}_{Q}$ and $\mathrm{k}_{F}^{Q}$ both scale as $\mathrm{n}_{Q}^{1 / 2}$ (the quasiparticle density), this means that $S_{x x}(1 / 2)$ and $\mathrm{S}_{x x}(3 / 2)$ are equal although $\mathrm{n}_{Q}$ differs by a factor of three between them. We also observe a $1 / n_{e}$ dependence for the CF TEP, while for the zero field TEP we observe rather something in between $1 / \mathrm{n}_{e}^{1.5}$ or $1 / \mathrm{n}_{e}^{2}$ corresponding to the fact that for zero field $m_{Q}$ is replaced by $m_{e}^{*}$, which is independent of the density.

\section{CONCLUSIONS}

We have demonstrated that the even denominator filling factor TEP can be described as the phonon drag TEP of CF's. All our experimental observations are explained by a zero field field expression for the phonon drag TEP of CF's in support of the thieoretical concept of these quasi-particles as fermions in zero field.

\section{REFERENCES}

[1] J. K. Jain, Adv. Phys. 41, 105 (1992).

[2] B. I. Halperin, P. A. Lee, and N. Read, Phys. Rev. B47, 7312 (1993).

[3] D. G. Cantrell and P. N. Butcher, J. Phys. C: Solid State Phys. 20, 1985 (1987); 20, 1993 (1987).

[4] B. Tieke et al., accepted for publication in Phys. Rev. Lett. (1996). 